\title{
The analysis of factors affecting price of selected commodities
}

\author{
Vladimíra Adamkovičová ${ }^{1, *}$, and Michal Gimerský ${ }^{1}$ \\ ${ }^{1}$ Technical University of Košice, Faculty of Economics, Němcovej 32, 04001 Košice, Slovakia
}

\begin{abstract}
Research background: Commodities are attractive investment opportunities and are objects of many researches. Traditional and most stable seem to be precious metals gold and silver. In the global world where anyone can access information in seconds it is crucial to know the background of price development in order to make the right investment decision.

Purpose of the article: This paper focuses on international commodity exchanges and analyzes factors affecting prices of selected commodities. It offers a summary of recent studies and continues with an analysis of most relevant factors affecting price of gold and silver.

Methods: After collecting data and testing their consistency we construct two hypothesis models. Using linear regression model we test the appropriateness of models which are constructed for each commodity. Subsequently, we test statistical significance of individual explanatory variables, such as dollar exchange rate, VIX, CPI, DJIA, DAX or oil price. Additionally, we take into consideration not traditional factors e.g. Bitcoin. Findings \& Value added: Finally an evaluation of results are carried out and the influence of variables are quantified. For instance, negative impact of VIX and dollar exchange rate in observed period of time was confirmed. Given the fact that there can be various other factors influencing price development of gold and silver this paper serves as basis for further analysis in this direction.
\end{abstract}

Keywords: gold; silver; commodity; regression analysis; econometric model

JEL Classification: $C 10 ; C 12 ; Q 02$

\section{Introduction}

Precious metals have been globally used as a means of exchange and store of value for millennia. Investment in gold or silver is possibility of diversification of an investment portfolio. This paper investigates if and to what extent factors influence prices of two selected commodities.

\footnotetext{
* Corresponding author: adamkovicova.vladimira@gmail.com
} 
The aim of the paper is to develop appropriate econometric models for the selected commodities and to analyze the impact of various factors on their prices. Over the last few decades, the prices of gold and silver have changed significantly due to a variety of measurable and non-measurable factors. We cannot deny significant events like 1996-1998, Dot-com crisis in 2001-2003, economic crisis in 2008-2010 or ongoing global COVID-19 pandemic. All of these events have a certain impact on the price of gold and silver, as they affect their supply and demand. There are not only significant events but also other factors influencing prices of gold and silver. Besides usual factors mentioned below, we add Bitcoin as the most famous globally recognized cryptocurrency and we measure its influence on both precious metals. (Jones and Sackley, 2014)

\section{Literature review}

There are many approaches how to address factors influencing gold and silver price. Probably the best summary of current researches provide Pierdzioch et al. (2014). According to this study, usually considered variables affecting gold price are Inflation rate, Interest rates, Index USD and Stock indices. Additionally, they cannot imply that gold markets are informationally efficient and suggest also to look into higher frequencies than monthly data. There are also researches studying gold as financial "safe heaven" (Baur et al., 2010, 2017). Here, an interesting insight in form of usage of gold-CPI and golddividend yield ratios is presented. An influence of CPI on gold is confirmed, suggesting that gold price tends to follow inflation after 6 months. They also analyzed impact of policy uncertainty on the relative price of gold. Their results indicated positive relationship which can serve as evidence of gold as safe haven. Negative impact was seen on gold-silver ratio, which means that there are some periods when silver is catching up with gold and thus confirm its characteristic as alternative safe haven. (Baur, 2017; Eryiğit, 2017)

The paper includes an analysis of the determinants that, according to the previous analyses studied, affect the prices of gold and silver. We add to our model also VIX index as also considered by Wei-Fong Pan (2017) and Bitcoin - called also "digital gold". A study of gold and Bitcoin as save haven instruments provide Selmi et al. (2018) however, they analyze gold and Bitcoin separately. Using quantile on quantile regression and the risk evaluation based on unconditional and conditional data analyses they proved that gold and Bitcoin in times of financial uncertainty have similar behavior. On the other hand, during times of economic stability, they behave dissimilarly. One of the recent studies from Qian et al. (2019) was investigating influence of six factors on the gold price: Dolar Index, Federal Funds Rate, CPI, Exchange rate, Oil price, S\&P500. Using Box-Behnken design a form of RSM conclude in the paper that the model sufficiently describes correlation between gold price and six chosen factors. Five of them had negative impact on gold prices and only CPI proved positive influence which is in line with studies of Levin and Wright (2006). They also believed in positive correlation between US CPI and gold price and that world inflation rate has not a strong influence. (Beckmann and Cudzaj, 2013)

To our knowledge, there is no research that focuses on influence of Bitcoin to gold prices. Al-Yahyaee et al. (2018) study efficiency of Bitcoin market in comparison with stock markets, where Bitcoin turn out to be inefficient in studied period of 2010-2017. Unfortunately, gold is not present in this research. Gajardo et al. (2018) run a research which shows that Bitcoin has bigger multifractal spectra than other currencies in cross correlation with gold.

Churchill et al. (2019) using parametric and non-parametric modeling are considering a long period of 135 years to examine relationship of oil price, precious metals and exchange rates US Dollar and British Pound. They find non-linearity and asymmetry in long-term relationship between oil and gold price and oil and silver price. Another study uses VECM 
model to investigate dynamics between gold price and real interest rate. Positive association between both variables was observed (Apergis et al., 2019).

\section{Methods}

\subsection{Methodology}

Based on monthly data obtained for different variables we build 2 econometric models and investigate the impact of selected factors on prices of chosen commodities using linear multivariate regression analysis. We will test appropriateness and statistical significance of both models and individual parameters as well. Using IBM SPSS Statistics software we will test distribution of residuals via Jarque-Bera test, heteroskedasticity via BreuschPagan test, autocorrelation via Durbin-Watson test and multicollinearity via Variance inflation factor (VIF) measure. Analysis of Variance (ANOVA) will show statistical significance of the model - if the result will be higher than significance level of 0,05 it will be necessary to adjust variables under consideration. Mostly used way of such adjustment is transformation to percentage changes, difference of two values and logarithm. (Dyhrberg, 2016)

Our econometric models are created to investigate if and to what extent chosen variables are affecting price of precious metals, in this case - gold and silver. In order to create suitable model we studied previous researches in this matter and selected independent variables for the following model:

$$
\begin{aligned}
P_{\text {gold }}=\beta_{0}+\beta_{1} & \cdot \text { IndexUSD }+\beta_{2} \cdot \text { CPI }_{U S}+\beta_{3} \cdot \text { Inflation }+\beta_{4} \cdot \text { CREDITrisk } \\
& +\beta_{5} \cdot \text { OilP }+\beta_{6} \cdot V I X+\beta_{7} \cdot E P U_{U S}+\beta_{8} \cdot E P U_{E U}+\beta_{9} \cdot S P 500 \\
& +\beta_{10} \cdot D J I A+\beta_{11} \cdot D A X+\beta_{12} \cdot \text { Bitcoin }+\varepsilon
\end{aligned}
$$

The same model will be used for explaining silver price development.

\subsection{Data}

After creating the above mentioned model we gathered monthly data with timespan from February 1992 to August 2021. In our model, two dependent (explained) variables are present - gold and silver prices. Gold prices are in USD in end-of-month value and are obtained from World Gold Council. Prices of silver were obtained from Quandl, also the end-of-month prices denominated in USD. The rest of variables are independent or explanatory variables and these were gathered from different public sources including investing.com, yahoo finance or Federal Reserve Bank of Saint Louis.

Following independent or explanatory variables will be analyzed in our models:

- IndexUSD - US dollar index - measure of U.S. dollar relative to the basket of 6 most significant trading partners for the US - Euro, Canadian Dollar, Japanese Yen, British Pound, Swiss Franc and Swedish Krona.

- $\mathrm{CPI}_{\mathrm{US}}$ - consumer price index in USA - a measure of the average change over time in the prices paid by urban consumers for a market basket of consumer goods and services.

- Inflation - percentual change in consumer prices at the end of chosen period in USA (in our case for one month period)

- CREDITrisk - credit risk default premium (CRDP) represents relation between Moody's Seasoned Corporate Bond Yield of Aaa and Baa rated bonds. 
- $\quad$ OilP - crude oil price WTI (in USD / barrel)

- VIX - CBOE volatility index - real time market index representing expectations of volatility over the coming 30 days, called also a fear index

- $\mathrm{EPU}_{\mathrm{US}}$ - Economic Policy Uncertainty in USA - constructed of 3 components: newspaper coverage of policy-related economic uncertainty, number of federal tax code provisions and disagreement among economic forecasters.

- $\quad \mathrm{EPU}_{\mathrm{EU}}$ - Economic Policy Uncertainty in EU

- $\quad$ SP500 - stock market index S\&P 500

- DJIA - stock market index Dow Jones Industrial Average

- DAX - most important German stock market index

- Bitcoin-cryptocurrency

As the obtained data covered periods with different timespan, it was necessary to find a reference period where all data of all variables will be available and complete. We proceeded to the data selection which resulted in database with 355 lines dating from February 1992 to August 2021. Second database was adapted to the period of Bitcoin trading history and thus this database contains 133 lines with each variable beginning with August 2010 until August 2021.

Our constructed econometric model mentioned in methodology will be carried out in 4 versions:

1) GOLD price model including variables except from Bitcoin (1992-2021)

2) GOLD price model including all variables (2010-2021)

3) SILVER price model including variables except from Bitcoin (1992-2021)

4) SILVER price model including all variables (2010-2021)

\section{Results}

\subsection{Gold Price Model}

Starting with the first model for gold including variables without Bitcoin and dataset starting from 1992 to 2021 we provided linear multivariate regression analysis using SPSS. First analysis showed us that we have a problem with multicollinearity, autocorrelation and heteroskedasticity.

For the purpose of this paper we show just correlation matrix (Table 1) for the period 2010-2021 in order to include all variables under consideration. Analysis showed that direction and significance in the longer period from 1992 prove the same tendency. Additionally, stock market indices DJIA, S\&P500 and DAX have all the same direction as the correlation between them is very high. 
Table 1. Correlation matrix - all variables in Gold Price Model.

\begin{tabular}{|c|c|c|c|c|c|c|c|c|c|c|c|c|c|c|}
\hline \multicolumn{15}{|c|}{ Correlations } \\
\hline & & Pgold & IndexUSD & CPIus & Inflation & CREDITrisk & OilP & VIX & EPUus & EPUeu & SP500 & DJIA & $\mathrm{DAX}$ & Bitcoin \\
\hline \multirow[t]{3}{*}{ Pgold } & Pearson Correlation & 1 & $-293^{* *}$ &, $262^{* \prime}$ &, $254^{-1}$ &, $505^{\prime \prime}$ & , 122 &, $479^{* *}$ &, $550^{\circ-1}$ &, $210^{\circ}$ &, $267^{*}$ &, $232^{*}$ &,- 015 &, $483^{*}$ \\
\hline & Sig. (2-tailed) & & ,001 & ,002 & ,003 & ,000 & 162 & ,000 & ,000 &, 015 &, 002 & ,007 & 866 & ,000 \\
\hline & $\mathrm{N}$ & 133 & 133 & 133 & 133 & 133 & 133 & 133 & 133 & 133 & 133 & 133 & 133 & 133 \\
\hline \multirow[t]{3}{*}{ IndexUSD } & Pearson Correlation & $-293^{*}$ & 1 & ,679"* &,- 120 & $269^{* 2}$ &,$- 885^{* \prime}$ &,- 057 &, $217^{\circ}$ &, $387^{* \prime}$ &, $637^{\circ}$ & ,648" &, $747^{*}$ &, $229^{* \prime}$ \\
\hline & Sig. (2-tailed) & ,001 & & ,000 & ,168 & ,002 & ,000 &, 516 & ,012 &, 000 &, 000 & ,000 & ,000 & ,008 \\
\hline & $\mathrm{N}$ & 133 & 133 & 133 & 133 & 133 & 133 & 133 & 133 & 133 & 133 & 133 & 133 & 133 \\
\hline \multirow[t]{3}{*}{ CPlus } & Pearson Correlation &, $262^{2-}$ & ,679" & 1 & , 166 & $268^{* \prime}$ &,$- 584^{* *}$ & ,077 &, $400^{*}$ &, $254^{* \prime}$ &, $978^{* \prime}$ &, $982^{* \prime \prime}$ & ,907* &, $731^{\prime \prime}$ \\
\hline & Sig. (2-tailed) &, 002 &, 000 & &, 056 &, 002 & ,000 &, 378 &, 000 &, 003 & ,000 &, 000 &, 000 &, 000 \\
\hline & $\mathrm{N}$ & 133 & 133 & 133 & 133 & 133 & 133 & 133 & 133 & 133 & 133 & 133 & 133 & 133 \\
\hline \multirow[t]{3}{*}{ Inflation } & Pearson Correlation &, $254^{\prime \prime}$ & -120 & , 166 & 1 &,$- 181^{*}$ & ,153 & $-201^{*}$ & \begin{tabular}{l|l|}
-129 \\
\end{tabular} &,$- 181^{\circ}$ &, $240^{\circ}$ &, $231^{\prime \prime}$ & $206^{\circ}$ & ,386" \\
\hline & Sig. (2-tailed) & ,003 & 168 & ,056 & & ,037 &, 078 & ,021 & ,138 &, 037 &, 005 & ,007 & ,017 & ,000 \\
\hline & $\mathrm{N}$ & 133 & 133 & 133 & 133 & 133 & 133 & 133 & 133 & 133 & 133 & 133 & 133 & 133 \\
\hline \multirow[t]{3}{*}{ CREDITrisk } & Pearson Correlation & ,505* & $269^{* *}$ & $268^{* \prime}$ &,$- 181^{\circ}$ & 1 & $-467^{* *}$ & ,608* &, $664^{* \prime}$ &, $427^{* *}$ &, $217^{\circ}$ &, $187^{\circ}$ & ,032 &, 121 \\
\hline & Sig. (2-tailed) &, 000 &, 002 &, 002 &, 037 & & ,000 &, 000 & ,000 &, 000 &, 012 & ,032 &, 716 & , 167 \\
\hline & $\mathrm{N}$ & 133 & 133 & 133 & 133 & 133 & 133 & 133 & 133 & 133 & 133 & 133 & 133 & 133 \\
\hline \multirow[t]{3}{*}{ OilP } & Pearson Correlation & ,122 &,$- 885^{* *}$ &,$- 584^{* \prime}$ & , 153 &,$- 467^{*}$ & 1 &,$- 195^{*}$ &,$- 400^{*}$ &,$- 437^{* *}$ &,$- 568^{* \prime}$ &,$- 555^{* 4}$ &,$- 634^{*}$ &,$- 232^{* *}$ \\
\hline & Sig. (2-tailed) &, 162 &, 000 &, 000 &, 078 & ,000 & &, 024 &, 000 &, 000 &, 000 &, 000 &, 000 &, 007 \\
\hline & $\mathrm{N}$ & 133 & 133 & 133 & 133 & 133 & 133 & 133 & 133 & 133 & 133 & 133 & 133 & 133 \\
\hline \multirow[t]{3}{*}{ VIX } & Pearson Correlation &, $479^{* *}$ & -,057 & ,077 &,$- 201^{\circ}$ & ,608" & $-195^{\circ}$ & 1 &, $658^{\circ+1}$ & $345^{* *}$ &, 030 & |001 &,$- 192^{*}$ & 137 \\
\hline & Sig. (2-tailed) & ,000 &, 516 &, 378 & ,021 & ,000 & ,024 & & ,000 & ,000 &, 728 & ,987 & ,027 & ,115 \\
\hline & $\mathrm{N}$ & 133 & 133 & 133 & 133 & 133 & 133 & 133 & 133 & 133 & 133 & 133 & 133 & 133 \\
\hline \multirow{3}{*}{ EPUus } & Pearson Correlation &, $550^{\circ-1}$ &, $217^{\circ}$ & ,400" &,- 129 & ,664" & $-400^{\circ-}$ & ,658" & 1 &, $582^{* *}$ &, $343^{\circ *}$ &, $329^{\circ-1}$ &, 154 &, $247^{\prime \prime}$ \\
\hline & Sig. (2-tailed) &, 000 &, 012 & 000 &, 138 &, 000 &, 000 &, 000 & &, 000 &, 000 &, 000 &, 077 &, 004 \\
\hline & $\mathrm{N}$ & 133 & 133 & 133 & 133 & 133 & 133 & 133 & 133 & 133 & 133 & 133 & 133 & 133 \\
\hline \multirow[t]{3}{*}{ EPUeu } & Pearson Correlation &, $210^{\circ}$ &, $387^{* *}$ & ,254" &,$- 181^{\circ}$ &, $427^{*}$ & $-437^{* *}$ & $345^{* \prime}$ & ,582* & 1 &, 165 &, $174^{\circ}$ & ,084 & ,004 \\
\hline & Sig. (2-tailed) &, 015 & ,000 & ,003 &, 037 & ,000 & ,000 &, 000 & ,000 & &, 057 & ,045 &, 336 & ,961 \\
\hline & $\mathrm{N}$ & 133 & 133 & 133 & 133 & 133 & 133 & 133 & 133 & 133 & 133 & 133 & 133 & 133 \\
\hline \multirow[t]{3}{*}{ SP500 } & Pearson Correlation &, $267^{* *}$ &, $637^{* *}$ & ,978* & $240^{* *}$ &, $217^{*}$ &,$- 568^{* *}$ & ,030 &, $343^{* *}$ & ,165 & 1 & ,991" & ,934* & $807^{*}$ \\
\hline & Sig. (2-tailed) & ,002 & ,000 & ,000 &, 005 & 012 & ,000 &, 728 & ,000 & ,057 & & ,000 & ,000 & ,000 \\
\hline & $\mathrm{N}$ & 133 & 133 & 133 & 133 & 133 & 133 & 133 & 133 & 133 & 133 & 133 & 133 & 133 \\
\hline \multirow[t]{3}{*}{ DJIA } & Pearson Correlation &, $232^{\prime \prime}$ &, $648^{* \prime}$ & $982^{*}$ &, $231^{*}$ &, $187^{\circ}$ &,$- 555^{* \prime}$ &,- 001 &, $329^{-1}$ &, $174^{\circ}$ & ,991" & 1 & ,934" &, $773^{* \prime}$ \\
\hline & Sig. (2-tailed) & ,007 & ,000 & ,000 & ,007 & ,032 & ,000 &, 987 & ,000 &, 045 &, 000 & & ,000 & ,000 \\
\hline & $\mathrm{N}$ & 133 & 133 & 133 & 133 & 133 & 133 & 133 & 133 & 133 & 133 & 133 & 133 & 133 \\
\hline \multirow[t]{3}{*}{$\mathrm{DAX}$} & Pearson Correlation & -,015 &, $747^{* *}$ & ,907"* & $206^{\circ}$ & ,032 &,$- 634^{* *}$ &,$- 192^{*}$ & ,154 & ,084 & ,934" & ,934"* & 1 &, $663^{*}$ \\
\hline & Sig. (2-tailed) &, 866 & ,000 & ,000 & ,017 &, 716 & ,000 &, 027 & ,077 & ,336 &, 000 & ,000 & & ,000 \\
\hline & $\mathrm{N}$ & 133 & 133 & 133 & 133 & 133 & 133 & 133 & 133 & 133 & 133 & 133 & 133 & 133 \\
\hline \multirow[t]{3}{*}{ Bitcoin } & Pearson Correlation &, $483^{* *}$ & $229^{* *}$ &, $731^{*}$ &, $386^{* \prime}$ & ,121 &,$- 232^{* \prime}$ & ,137 &, $247^{\circ *}$ & ,004 & $.807^{* \prime}$ &, $773^{* \prime}$ & ,663" & 1 \\
\hline & Sig. (2-tailed) & ,000 &, 008 & ,000 & ,000 & ,167 &, 007 & ,115 & ,004 & ,961 & ,000 & ,000 & ,000 & \\
\hline & $\mathrm{N}$ & 133 & 133 & 133 & 133 & 133 & 133 & 133 & 133 & 133 & 133 & 133 & 133 & 133 \\
\hline
\end{tabular}

Source: SPSS Statistics

For better understanding of statistical significance, ANOVA test was performed. Clearly, the model is significant and stable since the significance level which was set at $5 \%$ was not crossed as seen in Table 3. The value 0,905 of R-Squared (Table 2) indicates that the model was constructed correctly and with this model $90,5 \%$ of the total width of the gold price data can be explained by studied variables. However, Durbin-Watson test is not at optimal value of 2,0. Two variables have negative influence on gold price and five variables have positive impact. If the Index USD decreases by 10,571 points, price of gold increases by 1 point. When Oil price rises by 4,594 points, the price of gold rises by 1 point.

The final model is as follows:

$$
\begin{aligned}
P_{\text {gold }}=782,974 & -10,571 \cdot \text { IndexUSD }+1073,436 \cdot \text { CREDITrisk }+4,594 \cdot \text { OilP } \\
& -7,56 \cdot V I X+0,783 \cdot E P U_{U S}+2,025 \cdot E P U_{E U}+0,225 \cdot S P 500 \\
& +\varepsilon
\end{aligned}
$$


Table 2. Model Summary of Gold Price Model.

\begin{tabular}{|l|c|r|r|r|r|}
\hline Model & R & R Square & $\begin{array}{c}\text { Adjusted R } \\
\text { Square }\end{array}$ & $\begin{array}{c}\text { Std. Error of the } \\
\text { Estimate }\end{array}$ & Durbin-Watson \\
\hline 1 &, $952^{\mathrm{a}}$ &, 907 &, 905 & 159,75737 &, 423 \\
\hline
\end{tabular}

a. Predictors: (Constant), SP500, VIX, IndexUSD, EPUeu, OilP, CREDITrisk, EPUus

b. Dependent Variable: Pgold

Source: SPSS Statistics

Table 3. Gold Price Model - Analysis of Variance (ANOVA).

\begin{tabular}{|rl|r|r|r|r|r|}
\hline \multicolumn{1}{|l|}{ Model } & & Sum of Squares & \multicolumn{1}{|c|}{ df } & Mean Square & \multicolumn{1}{c|}{ F } & Sig. \\
\hline 1 & Regression & 86279782,268 & 7 & 12325683,181 & 482,936 &, $000^{\text {b }}$ \\
& Residual & 8856278,389 & 347 & 25522,416 & & \\
& Total & 95136060,657 & 354 & & & \\
\hline
\end{tabular}

a. Dependent Variable: Pgold

b. Predictors: (Constant), SP500, VIX, IndexUSD, EPUeu, OilP, CREDITrisk, EPUus

Source: SPSS Statistics

Table 4. Gold Price Model - coefficients of statistically significant variables.

\begin{tabular}{|c|c|c|c|c|c|c|c|c|}
\hline \multicolumn{9}{|c|}{ Coefficients $^{\mathrm{a}}$} \\
\hline & & \multicolumn{2}{|c|}{ Unstandardized Coefficients } & $\begin{array}{c}\text { Standardized } \\
\text { Coefficients }\end{array}$ & \multirow[b]{2}{*}{$\mathrm{t}$} & \multirow[b]{2}{*}{ Sig. } & \multicolumn{2}{|c|}{ Collinearity Statistics } \\
\hline \multicolumn{2}{|c|}{ Model } & B & Std. Error & Beta & & & Tolerance & VIF \\
\hline \multirow[t]{8}{*}{1} & (Constant) & 782,974 & 120,454 & & 6,500 &, 000 & & \\
\hline & IndexUSD & $-10,571$ & 1,259 &,- 209 & $-8,398$ & ,000 & ,433 & 2,311 \\
\hline & CREDITrisk & 1073,436 & 127,885 & ,224 & 8,394 & ,000 & ,377 & 2,655 \\
\hline & OilP & 4,594 & ,460 & ,255 & 9,983 & ,000 & , 410 & 2,439 \\
\hline & VIX & $-7,560$ & 1,501 &,- 115 & $-5,035$ & ,000 & ,514 & 1,944 \\
\hline & EPUus & ,783 & ,242 & ,093 & 3,230 & ,001 & ,323 & 3,100 \\
\hline & EPUeu & 2,025 & ,207 & ,261 & 9,768 &, 000 & ,375 & 2,665 \\
\hline & SP500 & ,225 & ,016 & ,359 & 14,243 & ,000 & ,422 & 2,370 \\
\hline
\end{tabular}

a. Dependent Variable: Pgold

\section{Source: SPSS Statistics}

As mentioned in the beginning of this paper we wanted to analyze also not traditional factors which could have influence on gold and silver prices. We considered Bitcoin - the cryptocurrency with biggest market capitalization as a potential driver of gold price. As a basis serves our previous final regression model with seven variables which we identified as significant. This model was extended with Bitcoin as new independent variable. The difference here is the tested period which in this case begins in 2010. VIX, Oil Price and EPUus did not show statistical significance, that is why the final model has only five variables. We proved that Bitcoin has very low but positive impact on gold prices. (Table 5). 
Table 5. Gold Price Model including Bitcoin - coefficients of statistically significant variables.

\begin{tabular}{|c|c|c|c|c|c|c|c|c|}
\hline \multicolumn{9}{|c|}{ Coefficients $^{\mathrm{a}}$} \\
\hline \multirow{2}{*}{\multicolumn{2}{|c|}{ Model }} & \multicolumn{2}{|c|}{ Unstandardized Coefficients } & $\begin{array}{c}\text { Standardized } \\
\text { Coefficients }\end{array}$ & \multirow[b]{2}{*}{$\mathrm{t}$} & \multirow[b]{2}{*}{ Sig. } & \multicolumn{2}{|c|}{ Collinearity Statistics } \\
\hline & & $\mathrm{B}$ & Std. Error & Beta & & & Tolerance & VIF \\
\hline \multirow[t]{6}{*}{1} & (Constant) & 2814,106 & 117,909 & & 23,867 &, 000 & & \\
\hline & IndexUSD & $-25,877$ & 1,862 &,- 924 & $-13,900$ &, 000 &, 327 & 3,061 \\
\hline & CREDITrisk & 1223,794 & 104,466 &, 500 & 11,715 &, 000 &, 795 & 1,259 \\
\hline & EPUeu & 1,046 &, 178 & ,263 & 5,868 &, 000 &, 721 & 1,387 \\
\hline & SP500 &, 159 &, 030 &, 552 & 5,271 &, 000 &, 132 & 7,593 \\
\hline & Bitcoin &, 004 & ,002 & , 188 & 2,272 & ,025 & ,211 & 4,734 \\
\hline
\end{tabular}

a. Dependent Variable: Pgold

Source: SPSS Statistics

\subsection{Silver Price Model}

For silver prices we carried out the same analysis and in comparison to gold we had to remove another 2 variables EPUus and VIX - see Table 8 with statistically significant variables and their coefficients. Also in this case Index USD has negative impact on the price - decreasing by 0,172 points will lead to increasing of silver price by 1 point. The other variables (Credit risk, Oil price, EPUeu and S\&P500) proved their positive influence on the price. $\mathrm{R}$ squared in this model fell down to $77,7 \%$ (Table 6) and the whole model was statistically significant (Table 7).

The final model is as follows:

$$
\begin{gathered}
P_{\text {silver }}=11,685-0,172 \cdot \text { IndexUSD }+6,587 \cdot \text { CREDITrisk }+0,150 \cdot \text { OilP } \\
+0,036 \cdot E P U_{E U}+0,002 \cdot S P 500+\varepsilon
\end{gathered}
$$

Table 6. Model Summary of Silver Price Model.

\begin{tabular}{|l|r|r|r|r|r|}
\hline \multicolumn{1}{|c|}{} & & Model Summary \\
Model & $\mathrm{R}$ & R Square & $\begin{array}{c}\text { Adjusted R } \\
\text { Square }\end{array}$ & $\begin{array}{c}\text { Std. Error of the } \\
\text { Estimate }\end{array}$ & Durbin-Watson \\
\hline 1 &, $883^{\mathrm{a}}$ &, 780 &, 777 & 4,1474795 &, 267 \\
\hline
\end{tabular}

a. Predictors: (Constant), SP500, IndexUSD, CREDITrisk, EPUeu, OilP

b. Dependent Variable: Psilver

Source: SPSS Statistics 
Table 7. Silver Price Model - Analysis of Variance (ANOVA).

\begin{tabular}{|c|c|c|c|c|c|c|}
\hline \multicolumn{7}{|c|}{ ANOVA $^{a}$} \\
\hline & & Sum of Squares & $\mathrm{df}$ & Mean Square & $\mathrm{F}$ & Sig. \\
\hline \multirow[t]{3}{*}{1} & Regression & 21288,519 & 5 & 4257,704 & 247,518 &, $000^{\mathrm{b}}$ \\
\hline & Residual & 6003,353 & 349 & 17,202 & & \\
\hline & Total & 27291,873 & 354 & & & \\
\hline
\end{tabular}

a. Dependent Variable: Psilver

b. Predictors: (Constant), SP500, IndexUSD, CREDITrisk, EPUeu, OilP

Source: SPSS Statistics

Table 8. Silver Price Model - coefficients of statistically significant variables.

\begin{tabular}{|c|c|c|c|c|c|c|c|c|}
\hline \multicolumn{9}{|c|}{ Coefficients $^{\mathrm{a}}$} \\
\hline \multirow{2}{*}{\multicolumn{2}{|c|}{ Model }} & \multicolumn{2}{|c|}{ Unstandardized Coefficients } & $\begin{array}{l}\text { Standardized } \\
\text { Coefficients }\end{array}$ & \multirow[b]{2}{*}{$\mathrm{t}$} & \multirow[b]{2}{*}{ Sig. } & \multicolumn{2}{|c|}{ Collinearity Statistics } \\
\hline & & $\mathrm{B}$ & Std. Error & Beta & & & Tolerance & VIF \\
\hline 1 & (Constant) & 11,685 & 3,107 & & 3,761 &, 000 & & \\
\hline & IndexUSD &,- 172 & 031 &,- 200 & $-5,510$ & 000 & ,477 & 2,097 \\
\hline & CREDITrisk & 6,587 & 2,653 & ,081 & 2,482 & 014 &, 590 & 1,696 \\
\hline & OilP &, 150 &, 012 & ,494 & 12,834 &, 000 & ,426 & 2,349 \\
\hline & EPUeu &, 036 & ,004 & 270 & 8,002 & 000 &, 552 & 1,810 \\
\hline & SP500 & 002 & 000 & , 185 & 5,303 & 000 &, 519 & 1,928 \\
\hline
\end{tabular}

a. Dependent Variable: Psilver

\section{Source: SPSS Statistics}

The last model was analogically to gold model extended with Bitcoin. Here only 4 variables remained in the final model (Index USD, Credit risk, EPUeu, Bitcoin), however Bitcoin has even lower impact on silver prices than in Gold model.

Table 9. Silver Price Model including Bitcoin - coefficients of statistically significant variables.

\begin{tabular}{|c|c|c|c|c|c|c|c|c|}
\hline \multicolumn{9}{|c|}{ Coefficients $^{\mathrm{a}}$} \\
\hline \multirow{2}{*}{\multicolumn{2}{|c|}{ Model }} & \multicolumn{2}{|c|}{ Unstandardized Coefficients } & $\begin{array}{l}\text { Standardized } \\
\text { Coefficients }\end{array}$ & \multirow[b]{2}{*}{ t } & \multirow[b]{2}{*}{ Sig. } & \multicolumn{2}{|c|}{ Collinearity Statistics } \\
\hline & & $\mathrm{B}$ & Std. Error & Beta & & & Tolerance & VIF \\
\hline \multirow[t]{5}{*}{1} & (Constant) & 86,029 & 3,737 & & 23,019 &, 000 & & \\
\hline & IndexUSD &,- 819 &, 046 &,- 943 & $-17,880$ &, 000 &, 791 & 1,265 \\
\hline & CREDITrisk & 11,491 & 3,995 &, 151 & 2,876 &, 005 & ,796 & 1,256 \\
\hline & EPUeu & ,024 &, 007 &, 195 & 3,543 &, 001 & ,726 & 1,378 \\
\hline & Bitcoin &, 000 & ,000 & ,222 & 4,562 &, 000 & ,929 & 1,077 \\
\hline
\end{tabular}

a. Dependent Variable: Psilver

Source: SPSS Statistics

\section{Conclusion}

In this paper we focused on factors potentially affecting gold and silver prices. Beginning with literature review and providing an overview of most recent studies we continued with designing and testing our econometric models using linear regression approach with multiple variables. After receiving data and testing for statistical significance in the SPSS 
software we adjusted our models in accordance with statistical significance of its parameters. Models had to be adjusted as there was a problem with high multicollinearity. We would also like to draw attention to the fact that collected data and outputs are to be carefully interpreted as standard normal distribution was not present in residuals.

Our study provides comparable results with previous studies in certain extent - we can confirm negative impact of Index USD on both studied precious metals gold an silver, but having weak impact on the latter. Our model proved that also VIX has a negative impact on gold prices but no significant impact on silver prices. On the other hand Credit risk has positive impact on gold and silver prices, which is in line with the theory. In comparison to other studies, variables Inflation and CPI were tested insignificant which is in contrast to other researches which observed positive relationship between these two parameters and gold and silver prices. It could be said that Oil price and both silver and gold prices move in the same direction. Furthermore, Economic policy uncertainty in general is affecting both precious metals, however, we find interesting that in case of silver only European Economic policy uncertainty index was tested as significant, the American index was removed from model as it lacked statistical significance. We can thus confirm findings of previous studies about characteristic of gold and silver as a safe haven for investors.

At the beginning we have chosen three important global indices S\&P500, DJIA and DAX. Those together caused problem with multicollinearity and two of them had to be removed from our models. The final models proved that S\&P500 is affecting both precious metals positively. Important research question was if the Bitcoin has influence on precious metals prices. Surprisingly, only a weak impact on both metals was observed.

Further researches are encouraged as there is much place for extending of parameters and using other techniques and methods in order to determine drivers of gold and silver price changes. This paper hoped to bring more insight and information about factors that affect global gold and silver prices in order to help investors and researchers to gain more knowledge about price development itself and attribute in decision making process in investment portfolio construction.

\section{References}

1. Pierdzioch, Ch., Risse, M., \& Rohloff, S. (2014). On the efficiency of the gold market: Results of a real-time forecasting approach. International Review of Financial Analysis 32(2014), 95-108.

2. Baur, D.G., \& Lucey, B.M. (2010). Is gold a hedge or a safe haven? An Analysis of Stocks, Bonds and Gold. Financial Review, 45, 217-229.

3. Wei-Fong Pan. (2017). Sentiment and asset price bubble in the precious metals markets. Finance Research Letters, 26(2018),106-111.

4. Selmi, R., Mensi, W., Hammoudeh, S., \& Bouoiyour, J. (2018). Is Bitcoin a hedge, a safe haven or a diversifier for oil price movements? A comparison with gold. Energy Economics, 74(2018), 787-801.

5. Qian, Y., Dan A. Ralescu, D. A., \& Zhang, B. (2019). The analysis of factors affecting global gold price. Resources Policy, 64(2019), 101478.

6. Levin, E., \& Wright, R. (2006). Short-run and long-run determinants of the price of gold. World Gold Council, 2006, 70.

7. Eryiğit, E. (2017). Short-term and long-term relationships between gold prices and precious metal (palladium, silver and platinum) and energy (crude oil and gasoline) prices. Economic Research-Ekonomska Istraživanja, 30(1), 499-510. 
8. Baur, D., Beckmann, J., \& Czudaj, R. (2017). The relative valuation of gold. Chemnitz Economic Papers, 005.

9. Al-Yahyaee, K., Mensi, W., \& Yoon, S. M. (2018). Efficiency, multifractality, and the long-memory property of the Bitcoin market: A comparative analysis with stock, currency, and gold markets. Finance Research Letters, 27(2018), 228-234.

10. Gajardo, G., Kristjanpoller, W. D., \& Minutolo, M. (2018). Does Bitcoin exhibit the same asymmetric multifractal cross-correlations with crude oil, gold and DJIA as the Euro, Grat British Pound and Yen?. Chaos, Solitons and Fractals, 109(2018), 195205.

11. Churchill, S. A., Inekwe, J., Ivanovski, K., \& Smyth, R. (2019). Dynamics of oil price, precious metal prices and the exchange rate in the long-run. Energy Economics, 84 (2019), 104508.

12. Apergis, N., Cooray, A., Khreief, N., \& Apergis, I. (2019). Do gold prices respond to real interest rates? Evidence from the Bayesian Markov Switching VECM model. Journal of International Financial Markets, Institutions \& Money, 60(2019), 134-148.

13. Su, Ch., Qin, M., Tao,R., \& Zhang, X. (2020). Is the status of gold threatened by Bitcoin?. Economic Research-Ekonomska Istraživanja, 33(1), 420-437.

14. Dyhrberg, AH. (2016). Hedging capabilities of bitcoin. Is is the virtual gold?. Finance Research Letters, 16(2016), 139-144.

15. Jones, T., \& Sackley, W. (2014). An uncertain suggestion for gold-pricing models: The effect of economic policy uncertainty on gold prices. Journal of Economics and Finance, 40(2), 1-13.

16. Beckmann, J., \& Czudaj, R. (2013) Gold as an inflation hedge in a time-varying coefficient framework. The North American Journal of Economics and Finance, 24 (2013), 208-222. 